\title{
CAMBIOS FISIOLÓGICOS Y MORFOLÓGICOS TEMPRANOS EN EL RIÑÓN REMANENTE DE DONANTES VIVOS
}

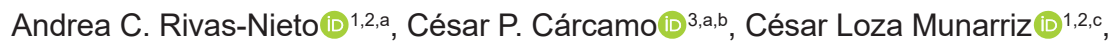 \\ Juan Miyahira $\mathbb{1}^{1,2, \mathrm{c}}$ \\ 1 Facultad de Medicina Alberto Hurtado, Universidad Peruana Cayetano Heredia, Lima, Perú. \\ 2 Servicio de Nefrología, Hospital Cayetano Heredia, Lima, Perú. \\ 3 Facultad de Salud Pública y Administración, Universidad Peruana Cayetano Heredia, Lima, Perú. \\ Médica/o cirujana/o; ${ }^{\mathrm{b}}$ doctor en Salud Pública; ${ }^{\mathrm{c}}$ médico nefrólogo
}

\section{RESUMEN}

Con el objetivo de describir los cambios funcionales y morfológicos tempranos en el riñón remanente de donantes vivos, se realizó un estudio retrospectivo en el Hospital Cayetano Heredia, en el que se incluyeron 55 individuos. De las historias clínicas, se obtuvieron los datos clínicos y demográficos, así como la depuración de creatinina, la proteinuria, la presión arterial y las dimensiones renales a los 1, 2, 3, 6 y 12 meses después de la donación del riñón. La edad media fue de 40,88 ( $\pm 9,84)$ años; el 80\% eran mujeres y el índice de masa corporal medio fue de $25,68( \pm 3,5) \mathrm{kg} / \mathrm{m}^{2}$. Se utilizaron modelos lineales y cuadráticos para estudiar las variables fisiológicas y morfológicas. Durante el tiempo de seguimiento, la tasa de filtración glomerular, la proteinuria, la presión arterial diastólica y la longitud de los riñones mostraron cambios significativos $(\mathrm{p}<0,05)$.

Palabras clave: Riñón; Riñón Único; Fisiología; Adaptación Fisiológica; Trasplante de Riñón; Donante de Tejido; Proteinuria; Presión Arterial; Tasa de Filtración Glomerular; Perú (Fuente: DeCS BIREME).

\section{EARLY PHYSIOLOGICAL AND MORPHOLOGICAL CHANGES IN THE REMNANT KIDNEY OF LIVING DONORS}

\begin{abstract}
In order to describe the early functional and morphological changes in the remnant kidney of living donors, a retrospective study was carried out at the Cayetano Heredia Hospital. Data from 55 individuals was included. Clinical and demographic data were obtained from the clinical records, as well as data for creatinine clearance, proteinuria, blood pressure and renal dimensions at 1, 2, 3, 6 and 12 months after kidney donation. The mean age was $40.88( \pm 9.84)$ years; $80 \%$ were women and the mean body mass index was $25.68( \pm 3.5) \mathrm{kg} / \mathrm{m}^{2}$. Linear and quadratic models were used to study physiological and morphological variables. During the follow-up time, glomerular filtration rate, proteinuria, diastolic blood pressure, and kidney length showed significant changes $(\mathrm{p}<0.05)$.
\end{abstract}

Keywords: Kidney; Solitary Kidney; Physiology; Physiological Adaptation; Kidney Transplantation; Tissue donor; Proteinuria; Arterial Pressure; Glomerular Filtration Rate; Peru (Source: MeSH NLM).

Citar como: Rivas-Nieto AC, Cárcamo CP, Loza Munarriz C, Miyahira J. Cambios fisiológicos y morfológicos tempranos en el riñón remanente de donantes vivos. Rev Peru Med Exp Salud Publica. 2020;37(4):7338. doi: https://doi.org/10.17843/ rpmesp.2020.374.5491.

Correspondencia: Andrea Cecilia Rivas Nieto; Av. Juan de Aliaga 488803, Magdalena del Mar, Lima, Perú; andrea.rivas@upch.pe

Recibido: $05 / 04 / 2020$ Aprobado: $16 / 07 / 2020$ En línea: 06/10/2020

\section{INTRODUCCIÓN}

La donación de un riñón implica en el donante vivo la pérdida abrupta de alrededor de la mitad de su función renal ${ }^{(1)}$. Por lo tanto, el riñón remanente de los donantes vivos experimenta cambios compensatorios tan pronto como en la primera semana luego de la nefrectomía y puede persistir hasta 10 años después ${ }^{(2,3)}$. Numerosos estudios de seguimiento de donantes en Europa, América, África y Asia describen que la función renal de un donante a largo plazo, con el adecuado seguimiento, es muy similar a la de una persona viviendo con ambos riñones ${ }^{(4-7)}$. Los parámetros más estudiados son la presión arterial (PA), la proteinuria, la tasa de filtración glomerular (TFG) y el riesgo para desarrollar enfermedad renal crónica estadio 5 (ERC5) ${ }^{(4-7)}$. Otros aspectos importantes para determinar el pronóstico de los pacientes son la edad de donación y el índice de masa corporal (IMC) basal. Los menores de 50 años tienen mejor pronóstico en la recuperación de la 
función renal luego de la nefrectomía ${ }^{(8)}$ y aquellos que presentan obesidad ( IMC $\geq 30$ ) carecen de reserva funcional renal tras la cirugía ${ }^{(9)}$.

La variación de la función renal refleja cambios en muchos parámetros clínicos de los donantes vivos. La TFG correspondiente al riñón remanente se incrementa desde la primera semana de seguimiento ${ }^{(10)}$. La PA muestra pocos cambios en el seguimiento a corto y largo plazo, manteniendo valores similares a los de antes de la cirugía con una baja incidencia de hipertensión arterial (HTA) ${ }^{(6,9,11)}$. La proteinuria es mínima luego de la nefrectomía, pero puede incrementarse a largo plazo y tiene mayor importancia debido a su asociación con la HTA y a la disminución de la tasa de filtración glomerular ${ }^{(11-13)}$. Por último, el riñón remanente experimenta cambios morfológicos desde la primera semana posnefrectomía. Se ha descrito un aumento significativo de la longitud, así como el incremento del volumen del riñón hasta $15 \%$ más a los tres meses ${ }^{(2,14)}$.

Si bien existen estudios en países nórdicos, que incluyen población hispana, sus autores coinciden en que sus resultados no necesariamente reflejan la realidad de los países latinoamericanos ${ }^{(15)}$. Debido a la inexistente información de seguimiento de donantes de riñón en población peruana y con la necesidad de conocer los cambios adaptativos morfológicos y fisiológicos tempranos del riñón remanente de los donantes vivos en el contexto etnográfico local, se realizó este estudio cuyo objetivo principal fue describir los cambios funcionales y morfológicos en el riñón remanente de donantes sanos durante el primer año luego de la nefrectomía.

\section{EL ESTUDIO}

Este estudio fue una serie de casos retrospectiva e incluyó a personas que habían donado un riñón entre 2007 y 2013, en el Programa de Trasplante Renal del Hospital Cayetano Heredia $(\mathrm{HCH})$. Dichas personas fueron evaluadas para descartar comorbilidades antes de ser aceptadas como donantes. Según las especificaciones del programa, los parámetros basales se obtienen, como máximo, tres meses antes de la nefrectomía. Una vez identificados los donantes efectivos y sus historias clínicas, se procedió a registrar y codificar los datos clínicos y demográficos de los pacientes en la ficha de recolección de datos elaborada específicamente para el estudio.

Se consideraron a las personas cuyas historias clínicas contenían datos basales y, como mínimo, un control durante el seguimiento posterior a la nefrectomía. Se excluyeron a aquellos participantes que tuvieran hipertensión arterial, diabetes mellitus, gestantes o cualquier condición clínica o fisiológica que pudiera influir en la evolución de la función renal.

Las variables del estudio fueron la TFG, medida con la depuración de creatinina en 24 horas corregida para una superficie corporal de $1,73 \mathrm{~m}^{2}$; la proteinuria medida en orina de 24 horas; la presión arterial sistólica (PAS) y la diastólica

\section{MENSAJES CLAVE}

Motivación para realizar el estudio: Conocer las variaciones de la función renal de los donantes vivos de riñón durante el primer año de seguimiento tras la nefrectomía, en el contexto peruano.

Principales hallazgos: Durante los doce primeros meses, la tasa de filtración glomerular, la proteinuria, la presión arterial diastólica y la longitud de los riñones mostraron cambios significativos. Dichos resultados fueron similares a otros estudios internacionales.

Implicancias: Este es el primer estudio que explora la adaptación fisiológica de los donantes de riñón peruanos y es el punto de partida para otras investigaciones relacionadas al tema.

(PAD); y las medidas del riñón (Longitud renal y grosor del parénquima) obtenidas mediante la ecografía realizada por un radiólogo experto. De manera exploratoria, se indagó sobre la ocurrencia de mortalidad por causa renal o cardiovascular y desarrollo de ERC5. Se recolectaron los datos basales y aquellos correspondiente al primer, segundo, tercer, sexto y décimo segundo mes de seguimiento. Se consideró la mitad de la TFG basal como aquella atribuible a un solo riñón del donante. Los datos fueron luego ingresados a una base de datos utilizando el programa Microsoft Excel para analizarse luego con el programa Stata versión 8.2.

\section{Análisis de datos}

Para las variables categóricas se determinaron frecuencias absolutas y relativas, y para las continuas, media y desviación estándar. A fin de describir la evolución de los parámetros clínicos observados, se construyeron modelos de regresión lineal, controlando por observaciones múltiples para un mismo participante. En el caso particular de la variable proteinuria, se hizo una transformación logarítmica para lograr una distribución normal. Se evaluaron términos lineales y cuadráticos para cada variable continua. Los supuestos de linealidad, independencia, homocedasticidad y normalidad se corroboraron para cada uno de los modelos finales obtenidos.

\section{Aspectos éticos}

Este estudio utilizó solo la información disponible en las historias clínicas y no implicó la toma de muestras o entrevistas directas a los donantes. La investigadora principal registró las fichas de recolección de datos en una computadora con contraseña, como medida de seguridad. Se asignó un código a cada participante para su vaciado en la base de datos por lo que otras personas no tuvieron acceso a datos personales. La Facultad de Medicina y el Comité Institucional de Ética de la Universidad Peruana Cayetano Heredia (Registro SIDISI 
61396), y el Servicio de Nefrología y el Comité de Ética del HCH (Código 096-013) revisaron y aprobaron el protocolo.

\section{HALLAZGOS}

Sesenta personas sanas donaron un riñón en el periodo 2007-2013. En el archivo del hospital se encontraron las historias clínicas de 56 donantes. Ninguna de ellas cumplió con los criterios de exclusión antes de los 12 meses de seguimiento. Finalmente, se incluyó a 55 donantes, de quienes se obtuvieron los datos basales. El promedio de edad fue 40,88 años (desviación estándar (DE): 9,84), el 80\% eran mujeres y el IMC basal promedio fue 25,68 (DE: 3,5). Al construir los modelos lineales y cuadráticos, se observó que la TFG y la proteinuria tenían un término cuadrático significativo. El resto de las variables permanecieron bajo un modelo lineal.

Se observó que la TFG disminuyó hasta el mes 6, para luego incrementarse al mes 12 (Figura 1A). Para las 14 personas con valores disponibles de TFG prequirúrgico y al año, la TFG promedio a los 12 meses $\left(94,1 \mathrm{~mL} / \mathrm{min} / 1,73 \mathrm{~m}^{2}\right)$ fue significativamente menor a la prequirúrgica $\left(114,5 \mathrm{~mL} / \mathrm{min} / 1,73 \mathrm{~m}^{2}\right.$, $\mathrm{p}=0,006)$, pero significativamente mayor a la mitad de este valor (TFG atribuible a un solo riñón) ( $p<0,001)$. Es decir, la TFG aumenta de forma significativa si se considera la TFG de un solo riñón como punto de inicio (Figura 1B).

La PAS no mostró cambios significativos a lo largo del tiempo (Figura 2A), no así la PAD, que tuvo un incremento significativo a lo largo del seguimiento (Figura 2B).

En cuanto a la proteinuria medida en orina de 24 horas, se observó aumento hasta el mes 6, para luego disminuir hasta el mes 12 , con cambios significativos a lo largo del seguimiento (Figura 3).

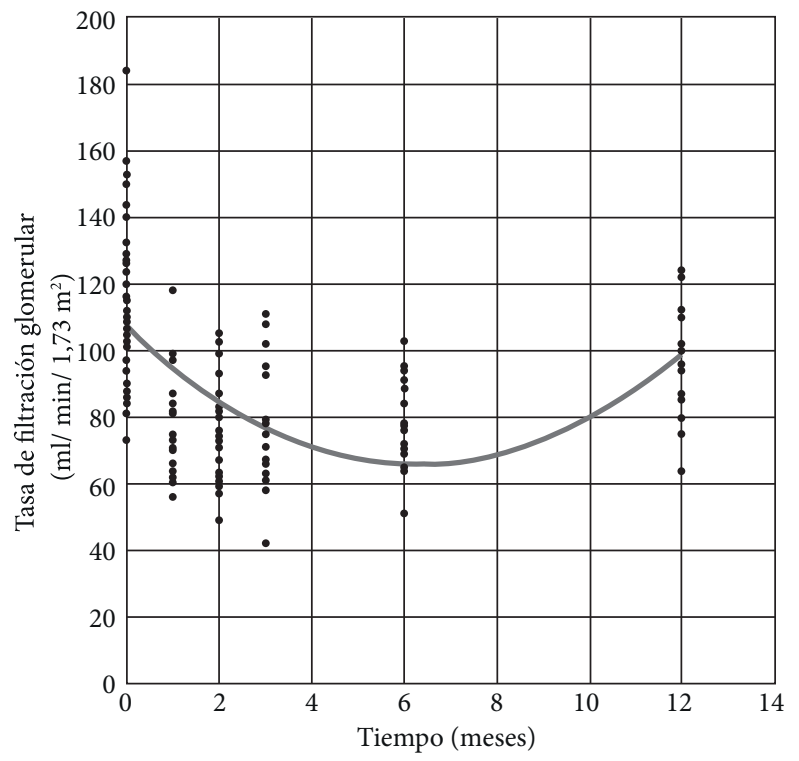

A
La longitud del riñón aumentó significativamente en el seguimiento (Figura 4A). Mientras que el parénquima renal no mostró un cambio significativo hasta los 12 meses (Figura 4B).

No se registraron defunciones durante el seguimiento. De los 14 donantes que registraron datos hasta el año, ninguno desarrolló ERC5. El menor valor registrado de TFG a los 12 meses fue $64 \mathrm{~mL} / \mathrm{min} / 1,73 \mathrm{~m}^{2}$.

\section{DISCUSIÓN}

En este grupo de donantes peruanos, la TFG, la proteinuria, la PAD y la longitud del riñón remanente mostraron cambios significativos durante el seguimiento. Los hallazgos coinciden con la información obtenida en la literatura. Un metaanálisis que incluyó numerosos estudios a nivel internacional encontró que la TFG global varía durante los primeros seis meses y es significativamente menor a la basal, incluso 10 años después ${ }^{(16)}$. Se observa que la TFG global disminuye hasta los seis meses $y$, aunque incrementa hasta el año de seguimiento, permanece significativamente menor al valor prequirúrgico. Sin embargo, tomando en cuenta solo el comportamiento de un riñón, se observa que la diferencia un año después sí es mayor.

La causa de la variación de la TFG en el riñón remanente es la hipertrofia renal posterior a la nefrectomía contralateral, la cual produce hiperfiltración a manera de compensación ${ }^{(17)}$. En este estudio, con los datos de las ecografías, se encontró que la longitud mayor del riñón remanente incrementa de manera significativa, lo que sería un indicador del aumento de la masa renal como consecuencia de la hipertrofia. Otros métodos, como la tomografía computari-

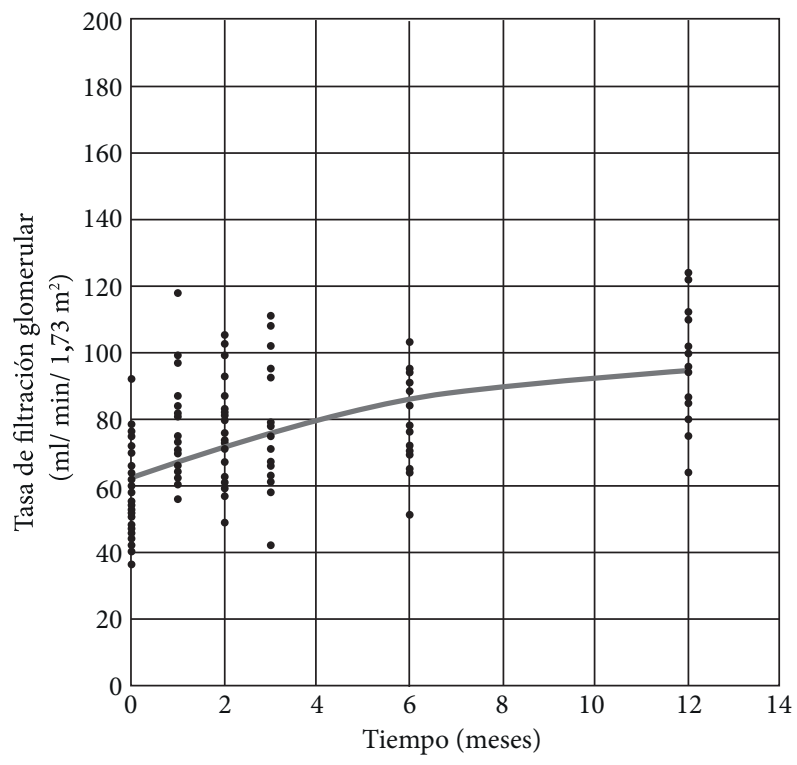

B

Figura 1. A: Tasa de filtración glomerular $\left(\mathrm{TFG}=106,742-13,057\right.$ mes $\left.+1,032 \mathrm{mes}^{2}\right)$. B: Tasa de filtración glomerular atribuible al riñón remanente. 

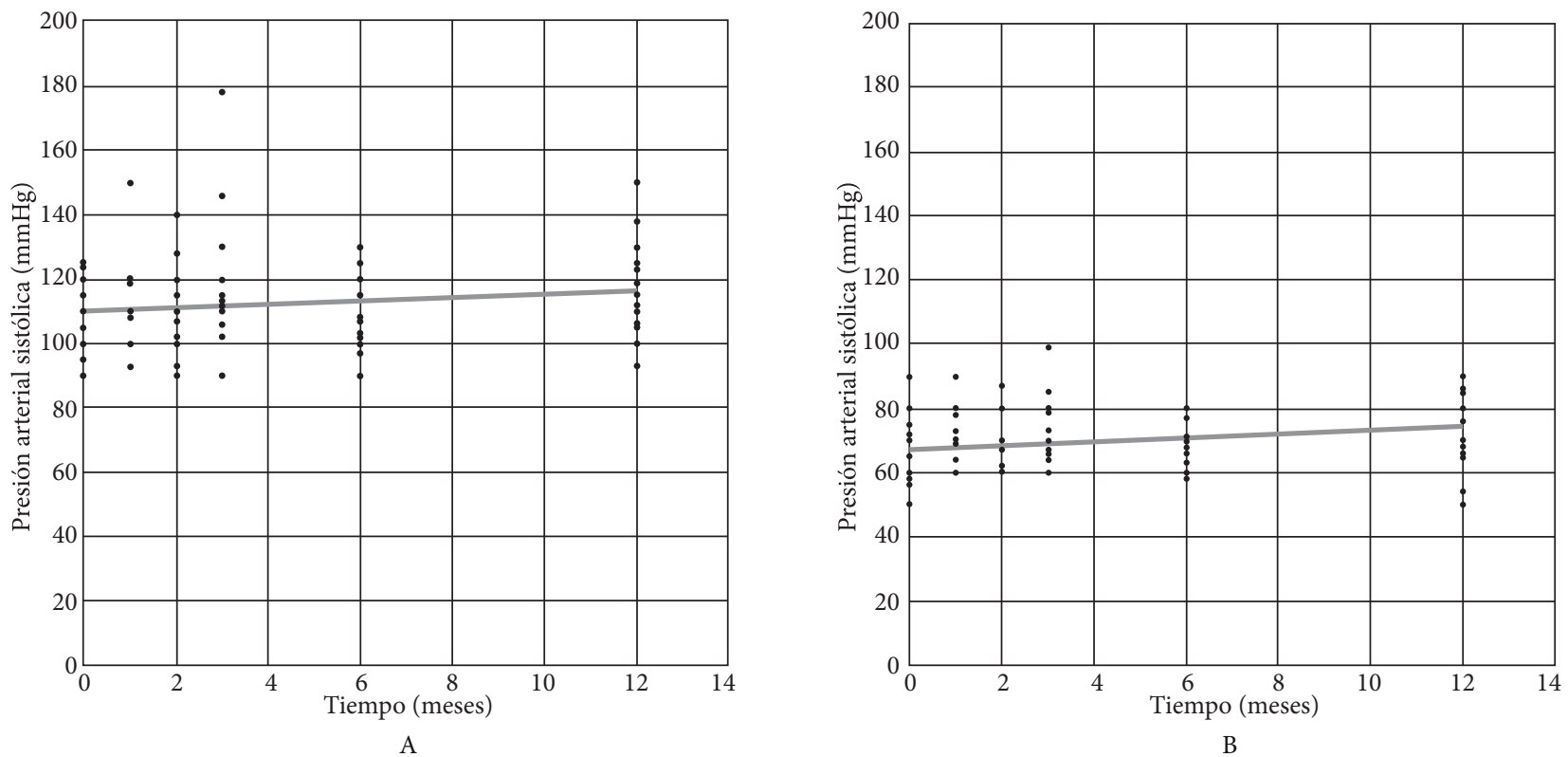

Figura 2. A: Presión arterial sistólica $(\mathrm{PAS}=109,881+0,503$ mes $)$; B: Presión arterial diastólica $(\mathrm{PAD}=67,137+0,608$ mes $)$.

zada (TC), son superiores a la ecografía y reducen el sesgo del operador. Dichas ventajas se muestran en un estudio que utilizó TC y encontró que la hipertrofia renal puede suceder tan pronto como a los 3 meses y continúa durante el primer año de seguimiento ${ }^{(14)}$.

Por otro lado, la proteinuria aumenta debido a la hiperfiltración y puede permanecer incrementada a largo plazo ${ }^{(16)}$. Este aspecto es importante en el seguimiento, ya que la proteinuria es un indicador de la progresión hacia enfermedad renal crónica y un factor de riesgo cardiovas-

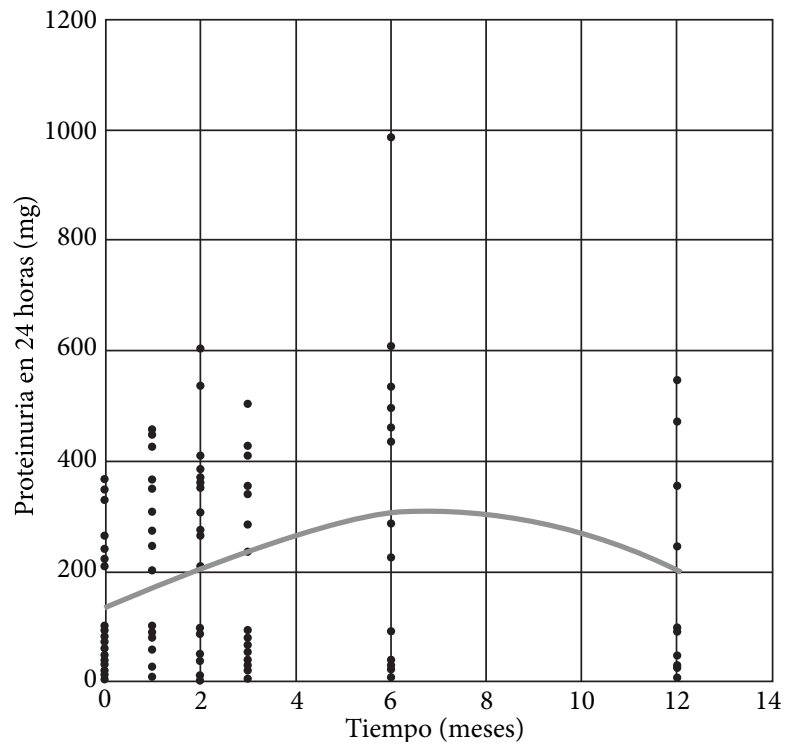

Figura 3. Proteinuria (Prot $\left.24 \mathrm{~h}=\mathrm{e}^{4,888+0,247 \text { mes }-0,018 \mathrm{mes} 2}\right)$. cular $^{(16)}$. En este grupo de donantes la proteinuria aumentó hasta los seis meses, descendiendo posteriormente, aunque no logró alcanzar niveles similares a los basales. Respecto a las variaciones de la PAS y de la PAD, la evidencia es heterogénea. Es esperable ver cambios en la presión arterial, ya que el sistema renina-angiotensina intrarrenal se activa en la primera semana posterior a la nefrectomía ${ }^{(18)}$.

En nuestro estudio, se pudo observar que solo la PAD se incrementa de forma significativa. Un metaanálisis realizado en el 2005, que evaluó estudios de comparación de seguimiento de donantes contra controles, mostró que los donantes tienen un incremento de $6 \mathrm{mmHg}$ en la PAS y $4 \mathrm{mmHg}$ en la PAD en un periodo de 5 a 10 años respecto a los controles. Estudios más recientes no observan variaciones en la presión arterial a los tres años ${ }^{(19)}$ o variaciones pequeñas, aunque significativas, sin llegar a niveles de HTA ${ }^{(20)}$. Se requiere ampliar el estudio de la variación de la presión arterial en donantes en poblaciones más grandes de donantes.

Entre otros factores que pueden influir en la recuperación de la función renal, resaltan la edad y el IMC. Los donantes más jóvenes tienen mayor desarrollo de hiperfiltración tras la nefrectomía que los mayores de 50 años ${ }^{\left({ }^{8}\right)}$. La población estudiada en este trabajo tenía una edad promedio de 40 años, por lo que era esperable que la recuperación sea adecuada. Por otro lado, el IMC es un factor condicionante para la recuperación de la función renal ${ }^{(9)}$. En este caso, la población tuvo un promedio de IMC de 25, lo que la sitúa en el límite inferior del sobrepeso. Si bien se encontraron donantes con IMC de hasta 35, el estudio no contó con datos adecuados para realizar análisis exploratorios en este aspecto. 


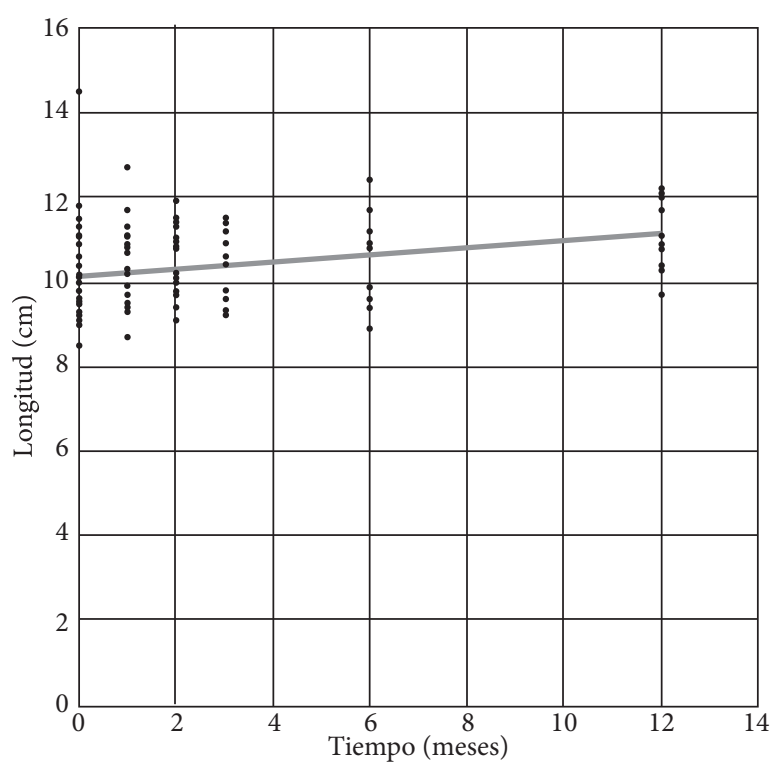

A

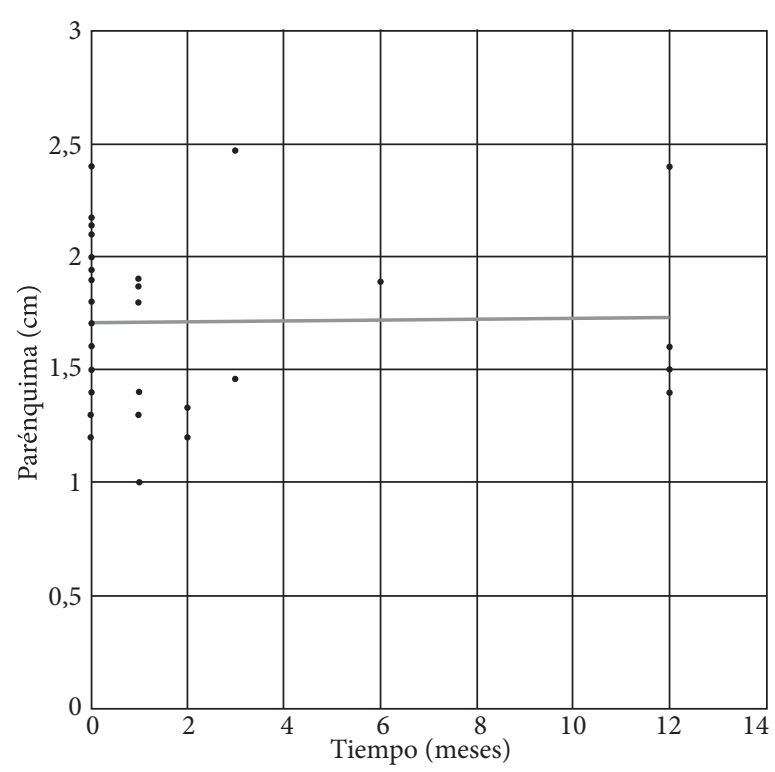

$\mathrm{B}$

Figura 4. A: Longitud del riñón (Long = 10,149 + 0,081 mes); B: Parénquima del riñón (Long = 1,708 + 0,001 mes).

En términos generales, los donantes vivos de riñón tienen una esperanza de vida y un riesgo de tener HTA y ERC5 similar a los de la población general ${ }^{(6)}$. La mayoría de los estudios de este tipo se han realizado en países de altos ingresos ${ }^{(16)}$. En un estudio realizado en los Estados Unidos, que analizó la probabilidad de desarrollar ERC5 en un grupo de donantes vivos sanos, se observó que la población hispana tuvo mayor riesgo que la caucásica, pero menor que la afroamericana. No obstante, los autores advierten que no necesariamente puede trasladarse a otras áreas geográficas ${ }^{(15)}$, por lo que no reflejaría la realidad de la región sudamericana y del Perú por su diversidad étnica y características sociodemográficas. Por ello, es imprescindible que toda institución peruana que realice trasplante renal tenga un registro y seguimiento adecuado de los donantes, a fin de tener información certera de la población local.

Una de las limitaciones de este estudio fue la falta de información o los datos incompletos en las historias clínicas, que restringió la exploración de eventos como la ERC. Lo ideal sería realizar un estudio prospectivo para un mejor análisis de las variables que pueden influir en la adaptación renal. Sin embargo, este es el primer esfuerzo por sistematizar la información de los donantes de un hospital público y se espera que trascienda a otras instituciones que realicen trasplante de órganos provenientes de donantes vivos.

En conclusión, en esta población la TFG del riñón remanente, la proteinuria, la $\mathrm{PAD}$ y la longitud del riñón remanente mostraron un incremento significativo a lo largo del primer año después de la nefrectomía contralateral.

Contribución de los autores: ACRN, JM y CL diseñaron la metodología de este estudio. CPC y ACRN concibieron y diseñaron el análisis. ACRN y JM recogieron los datos. CPC, CL y ACRN realizaron el análisis estadístico. ACRN, CPC, CL y JM redactaron el artículo.

Financiamiento: Autofinanciado.

Conflictos de interés: Los autores declaran no tener conflictos de interés.

\section{REFERENCIAS BIBLIOGRÁFICAS}

1. Delanaye P, Weekers L, Dubois BE, Cavalier E, Detry O, Squifflet J-P, et al. Outcome of the living kidney donor. Nephrol Dial Transplant. 2012;27(1):41-50. doi: 10.1093/ndt/gfr669.

2. Anderson RG, Bueschen AJ, Lloyd LK, Dubovsky EV, Burns JR. ShortTerm and Long-Term Changes in Renal Function After Donor Nephrectomy. J Urol. 1991;145(1):11-13. doi: 10.1016/S0022-5347(17)38232-0.

3. Chang S-G, Kim J-H, Lee S-J, Choi J-M, Huh J-S. Factors influencing contralateral renal hypertrophy after living donor nephrectomy. Trans- plant Proc. 2002;34(4):1139-42. doi: 10.1016/S0041-1345(02)02762-8.

4. El-Agroudy AE, Sabry AA, Wafa EW, Neamatalla AH, Ismail AM, Mohsen $\mathrm{M}$, et al. Long-term follow-up of living kidney donors: a longitudinal study. BJU Int. 2007;100(6):1351-1355. doi: 10.1111/j.1464-410X.2007.07054.x.

5. Fournier C, Pallet N, Cherqaoui Z, Pucheu S, Kreis H, Méjean A, et al. Very long-term follow-up of living kidney donors: Living kidney donors in the very long term. Transpl Int. 2012;25(4):385-390. doi: 10.1111/j.14322277.2012.01439.x. 
6. Ibrahim HN, Foley R, Tan L, Rogers T, Bailey RF, Guo H, et al. Long-Term Consequences of Kidney Donation. N Engl J Med. 2009;360(5):459-469. doi: 10.1056/NEJMoa0804883.

7. Oppenheimer Salinas F. Short, medium and long-term follow-up of living donors. Nefrologia. 2010;30 Suppl 2:100-5. doi: 10.3265/Nefrologia. pre2010.Nov.10699.

8. Fehrman-Ekholm I, Kvarnström N, Söfteland JM, Lennerling A, Rizell $\mathrm{M}$, Odén A, et al. Post-nephrectomy development of renal function in living kidney donors: a cross-sectional retrospective study. Nephrol Dial Transplant. 2011;26(7):2377-2381. doi: 10.1093/ndt/gfr161.

9. Rook M, Bosma RJ, van Son WJ, Hofker HS, Homan van der Heide JJ, ter Wee PM, et al. Nephrectomy Elicits Impact of Age and BMI on Renal Hemodynamics: Lower Postdonation Reserve Capacity in Older or Overweight Kidney Donors. Am J Transplant. 2008;8(10):2077-2085. doi: 10.1111/j.1600-6143.2008.02355.x.

10. Oh CK, Yoon SN, Lee BM, Kim JH, Kim SJ, Kim K, et al. Beneficial Effects on the Renal Function of Both Recipients and Donors in Living Donor Kidney Transplantation. Transplant Proc. 2008;40(7):2310-2312. doi: 10.1016/j.transproceed.2008.07.001.

11. Borchhardt KA, Yilmaz N, Haas M, Mayer G. Renal function and glomerular permselectivity late after living related donor transplantation. Transplantation. 1996;62(1):47-51. doi: 10.1097/00007890-19960715000010 .

12. Garg AX, Muirhead N, Knoll G, Yang RC, Prasad GVR, Thiessen-Philbrook $\mathrm{H}$, et al. Proteinuria and reduced kidney function in living kidney donors: A systematic review, meta-analysis, and meta-regression. Kidney Int. 2006;70(10):1801-1810. doi: 10.1038/sj.ki.5001819.
13. Goldfarb DA, Matin SF, Braun WE, Schreiber MJ, Mastroianni B, Papajcik D, et al. Renal outcome 25 years after donor nephrectomy. J Urol. 2001;166(6):2043-2047.

14. Shehab AB, Shaheen FA, Fallatah A, Al-Jobori AG, Sheikh IA, Al-Koussi M. Early changes in volume and function of the remaining kidney after unilateral donor nephrectomy. Saudi J Kidney Dis Transpl. 1994;5(4):474-478.

15. Muzaale AD, Massie AB, Wang M-C. Risk of end-stage renal disease following live kidney donation. JAMA. 2014;311(6):579-586. doi: 10.1001/ jama.2013.285141.

16. Li S-S, Huang Y-M, Wang M, Shen J, Lin BJ, Sui Y, et al. A meta-analysis of renal outcomes in living kidney donors. Medicine (Baltimore). 2016;95(24):e3847. doi: 10.1097/MD.0000000000003847.

17. Chen KW, Wu MWF, Chen Z, Tai BC, Goh YSB, Lata R, et al. Compensatory Hypertrophy After Living Donor Nephrectomy. Transplant Proc. 2016;48(3):716-719. doi: 10.1016/j.transproceed.2015.12.082.

18. Ohashi N, Isobe S, Matsuyama T, Ishigaki S, Suzuki T, Tsuji T, et al. The Intrarenal Renin-angiotensin System Is Activated Immediately after Kidney Donation in Kidney Transplant Donors. Intern Med Tokyo Jpn. 2019;58(5):643-648. doi: 10.2169/internalmedicine.1756-18.

19. Kasiske BL, Anderson-Haag T, Israni AK, Kalil RS, Kimmel PL, Kraus ES, et al. A prospective controlled study of living kidney donors: threeyear follow-up. Am J Kidney Dis. 2015;66(1):114-124. doi: 10.1053/j. ajkd.2015.01.019.

20. Kim SH, Hwang HS, Yoon HE, Kim YK, Choi BS, Moon IS, et al. Longterm risk of hypertension and chronic kidney disease in living kidney donors. Transplant Proc. 2012;44(3):632-634. doi: 10.1016/j.transproceed.2011.12.066 